\title{
Anti-Epileptic Medication Exposure Influences Functional Status in New Zealand Stroke Patients: A Retrospective Population-Level Study
}

\author{
Ailsa L. McGregor ${ }^{1}$ (D $\cdot$ Md. Rashedul Hoque ${ }^{2,3} \cdot$ Sophia Nickel $^{1} \cdot$ Alesha J. Smith ${ }^{1} \cdot$ Mohammad Atiquzzaman $^{4}$
}

Accepted: 31 August 2021 / Published online: 27 September 2021

(c) The Author(s) 2021

\begin{abstract}
Background Patients who develop seizures after stroke have disproportionately poorer outcomes and increased mortality. Objective Our objective was to investigate whether exposure to anti-epileptic medications influenced long-term functional status after stroke.

Methods We used linked health administrative data from a cohort of adult stroke patients in New Zealand. Demographics and prescription information were obtained from the National Minimum Dataset and Pharmaceutical Collection, respectively. Activities of daily living (ADL) scores for the same patients were obtained using the International Resident Assessment Instrument. Beta regression was used to investigate the relationship between anti-epileptic drug (AED) exposure and functional status.

Results The study included 3606 patients with a single ischaemic stroke between 2012 and 2017. In total, 15\% were dispensed an AED in the 3 months before or after stroke. The adjusted odds ratio (OR) for AED exposure was 1.29 (95\% confidence interval [CI] 1.15-1.45). Overall AED exposure, categorical body mass index (BMI), ethnicity, length of hospital stay, and exposure to paracetamol, opioids, anti-psychotics, and anti-nausea medications were significantly associated with changes in the mean ADL score percentages. Considering the exposure timeframe, the ORs for AED exposure only after stroke and for exposure both before and after stroke were 1.52 (95\% CI 1.31-1.78) and 1.09 (95\% CI 0.93-1.27), respectively.

Conclusion Stroke patients with AED exposure had greater odds of a higher ADL score, indicating a poorer long-term functional status than those unexposed to AEDs. The timeframe of exposure impacted on functional status, with patients exposed only after stroke having increased odds of higher ADL scores than those exposed both before and after stroke.
\end{abstract}

Ailsa L. McGregor

ailsa.mcgregor@otago.ac.nz

1 School of Pharmacy, Division of Health Sciences, University

of Otago, PO Box 56, Dunedin 9054, New Zealand

2 Faculty of Health Sciences, Simon Fraser University, Burnaby, Canada

3 Arthritis Research Canada, Vancouver, Canada

4 British Columbia Renal Agency, Vancouver, BC, Canada 


\section{Key Points}

Anti-epileptic drug exposure is associated with poorer long-term functional status after stroke.

Patients who develop epilepsy after stroke have higher odds of a poor functional status after stroke than patients with epilepsy who subsequentlysuffer a stroke.

Understanding how commonly prescribed medicines affect functional status may enable healthcare professionals to reduce the number of patients living with severe stroke-related deficits.

\section{Introduction}

Epilepsy after stroke is a significant clinical issue affecting approximately $7 \%$ of patients aged $>65$ years [1]. Poststroke epilepsy is associated with poor prognosis and increased mortality [2,3].

No consensus or clinical guidelines on the management of seizures following ischaemic stroke currently exist [4, 5]. There is little evidence to support seizure prevention [6], and prophylactic administration of anti-epileptic drugs (AEDs) has been related to poorer outcomes in other neurological conditions [7]. Administering AEDs after the first seizure decreases seizure recurrence [8], but preclinical evidence suggests that AED exposure post-stroke hinders functional recovery $[9,10]$. The impact of AEDs on functional recovery may relate to the generation of the drug and the timeframe of administration in relation to stroke. Topiramate, a second-generation AED, displayed neuroprotective properties and improved motor behaviour in a preclinical animal model when administered early after stroke [11]; however, phenytoin, a first-generation AED, was detrimental to motor function when administered after, but not before, stroke [12]. A review by Ryvlin et al. [13] extended this premise and suggested that firstgeneration AEDs such as phenytoin should be avoided in post-stroke epilepsy because of their detrimental effect on functional recovery and that new-generation drugs, including lamotrigine or gabapentin, are preferred options for first-line management because of their improved pharmacokinetic and pharmacodynamic profiles [13]. In contrast, sodium valproate, another first-generation AED, decreased neuronal damage and improved functional outcome following experimental status epilepticus [14] and promoted functional recovery in a preclinical stroke model [15]. Taken together, this evidence suggests that different generations of AEDs, and indeed different AEDs within a generation, can have significantly different effects on functional outcome.

The complex relationship between AED exposure and stroke outcome provides the potential to increase patient gains by managing the prescribing of potentially detrimental drugs. The objective of this study was to investigate any association between exposure to AEDs and functional status assessed using the activities of daily living (ADL) score in New Zealand stroke patients.

\section{Methods}

\subsection{Study Population}

We used linked health administrative data from a population-based cohort of 5640 stroke patients from New Zealand. A case definition of at least one hospital admission for ischaemic stroke (International Classification of Diseases, Tenth Edition code I60-I69) from 1 January 2012 to 31 December 2017, regardless of age, was adopted to identify patients in the national minimum dataset (NMDS). Patients were removed from the dataset if they experienced more than one stroke in the study period. Variables included age, sex, ethnicity, date of hospital admission, and length of hospital stay (LOS). The first admission date was considered the stroke diagnosis date. Prescription dispensing records in the 90 days before and 90 days after the stroke diagnosis date were obtained from the Pharmaceutical Collection and linked for each patient. The NMDS and Pharmaceutical Collection capture hospitalisation information and community dispensed prescription medicines for approximately $96 \%$ of New Zealanders. Functional status was assessed using a standardised ADL score extracted from the International Resident Assessment Instrument (interRAI ${ }^{\mathrm{TM}}$ ) and linked for each patient using national health index (NHI) numbers (a unique health identifier used for a patient's lifetime across all aspects of the health system). The National Ethics Advisory Committee's ethical guidelines permit the re-use of de-identified Ministry of Health data for research with a written consent waiver. 


\subsection{Outcome Variable}

Total ADL score (range 0-60) was the primary outcome; this is a summed score from ten subscales (bathing, personal hygiene, dressing upper body, dressing lower body, walking, locomotion, transfer toilet, toilet use, bed mobility, and eating). Scores for each subscale ranged from 0 (independent) to 6 (completely dependent) or 8 (the activity did not occur). For scores of 8, total dependence was assumed [16].

Patients with a functional assessment date only prior to their stroke diagnosis date were removed. Only the first assessment after stroke diagnosis date was considered.

\subsection{Anti-Epileptic Drug (AED) Exposure Definition}

The independent variable was exposure to AEDs. The AED variable included all medications in the dataset approved for epilepsy management in New Zealand (see Table 1 in the electronic supplementary material [ESM]). Using Pharmaceutical Collection data, a binary variable for overall AED exposure was created. Patients were considered exposed if they were dispensed an AED prescription any time in the 90 days before and 90 days after stroke diagnosis date. AED exposure was further categorised as follows:

A

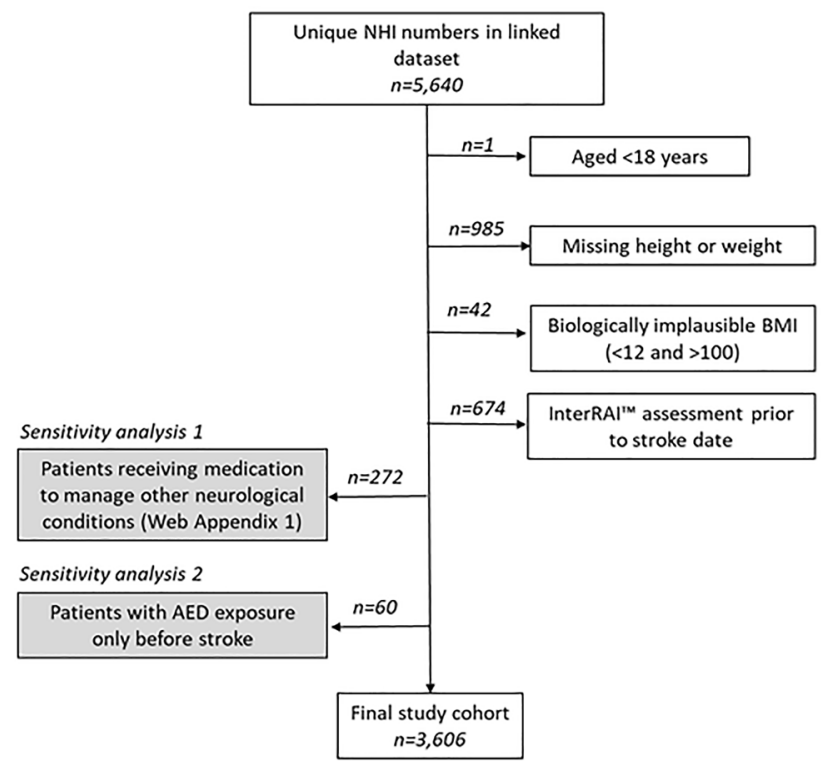

Fig. 1 A total of 3606 patients were included in the study. The patient populations used to conduct sensitivity analyses are indicated in the shaded area on the left of the figure (A). The median total ADL score was higher in patients exposed to AEDs only after stroke, and scores in the upper quartile varied less compared with other exposures. The
- 'after stroke'-patient dispensed an AED $\leq 90$ days after stroke

- 'both before and after stroke' - patient dispensed an AED both in the 90 days preceding and in the 90 days after stroke

- 'unexposed'-patient not dispensed an AED during this period (reference group).

We excluded patients exposed to AEDs only before their stroke in the primary analysis.

\subsection{Covariates}

Biological variables associated with functional ability were included [17]. Age in years on stroke diagnosis date was categorised into $16-64,65-74,75-84$, and $\geq 85$ years. Body mass index (BMI) was derived from height and weight measurements in interRAI ${ }^{\mathrm{TM}}$. Patients with missing height or weight measurements or a calculated BMI outside the range 12-100 were excluded (Fig. 1A). BMI was categorised as underweight $(<18.50)$, normal weight $(18.50-24.99)$, overweight (25.00-29.99), and obese $(\geq 30.00)$, consistent with New Zealand thresholds [18]. Sex was categorised as male or female and ethnicity as European, Māori, Pacific people, Asian, and other.

B

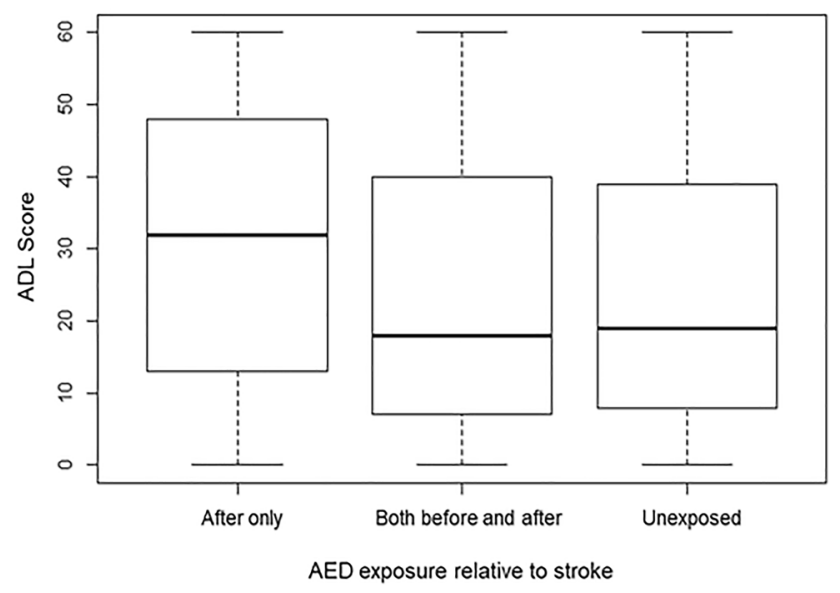

median ADL score and interquartile range in patients exposed both before and after stroke was comparable to that of unexposed patients (B). $A E D$ anti-epileptic drug, $A D L$ activities of daily living, $B M I$ body mass index, $N H I$ national health index, interRAI ${ }^{\mathrm{TM}}$ international residential assessment instrument 
Both initial stroke severity and how long after stroke a patient is assessed influence ADL scores. As such, LOS, a surrogate for initial stroke severity $[19,20]$, and time to assessment were included.

Binary exposure to a range of other centrally acting medications that may affect function post-stroke were included [21] (see Table I in the ESM). Patients who received medication for Parkinson's disease, Huntington's disease, or dementia or who received aprepitant to manage post-operative nausea and nausea related to chemotherapy were also excluded.

The Human Ethics Committee at the University of Otago, New Zealand, reviewed and approved this study (reference: HD17/065). All inferences, opinions, and conclusions drawn in this article are those of the authors and do not reflect the opinions or policies of the data steward(s).

\subsection{Statistical Analysis}

Baseline characteristics for AED-exposed and -unexposed stroke patients were compared using chi-squared tests for categorical variables or Wilcoxon's rank-sum tests for continuous variables.

To account for the non-normal and highly skewed distribution of ADL scores (Fig. I in the ESM), beta regression was used to investigate the relationship between exposure to AED and functional status in New Zealand stroke patients. The model was adjusted for sex and age category, categorical BMI, ethnicity, LOS, time to assessment, and exposure to medications that may affect post-stroke functional ability, including non-steroidal anti-inflammatory drugs, paracetamol, opioids, anti-depressants, anti-psychotics, hypnotics, anti-muscarinics, and anti-nausea, migraine, and smoking cessation medications. Since the beta regression outcome needs to be in the range $(0,1)$, ADL scores were expressed as a ratio of the total possible score of 60 for each patient.

Table 1 Population characteristics

\begin{tabular}{|c|c|c|c|c|}
\hline \multirow[t]{2}{*}{ Variable } & \multirow[t]{2}{*}{ Total sample $(n=3606)$} & \multicolumn{3}{|l|}{ AED exposure category } \\
\hline & & Unexposed $(n=3058)$ & Exposed $(n=548)$ & $p$ value \\
\hline \multicolumn{5}{|l|}{ Age category (years) } \\
\hline $16-64$ & $172(4.8)$ & $121(4.0)$ & $51(9.3)$ & \multirow{4}{*}{$<0.001$} \\
\hline $65-74$ & $594(16.5)$ & $471(15.4)$ & $123(22.5)$ & \\
\hline $75-84$ & $1385(38.4)$ & $1172(38.3)$ & $213(38.9)$ & \\
\hline$\geq 85$ & $1455(40.4)$ & $1294(42.3)$ & $161(29.4)$ & \\
\hline \multicolumn{5}{|l|}{ Sex } \\
\hline Male & $2139(59.3)$ & $1839(60.1)$ & $300(54.7)$ & \multirow[t]{2}{*}{0.018} \\
\hline Female & $1467(40.7)$ & $1219(40.2)$ & $248(45.3)$ & \\
\hline \multicolumn{5}{|l|}{ Ethnic category } \\
\hline European & $3127(86.7)$ & $2657(86.9)$ & $470(85.8)$ & \multirow{5}{*}{0.007} \\
\hline Māori & $184(5.1)$ & $143(4.7)$ & $41(7.5)$ & \\
\hline Pacific people & $116(3.2)$ & $96(3.1)$ & $20(3.7)$ & \\
\hline Asian & $114(3.2)$ & $100(3.3)$ & $14(2.5)$ & \\
\hline Other ethnicity & $65(1.8)$ & $62(2.0)$ & $3(0.6)$ & \\
\hline \multicolumn{5}{|l|}{ BMI category } \\
\hline Underweight & $279(7.7)$ & $239(7.8)$ & $40(7.3)$ & \multirow{4}{*}{0.007} \\
\hline Normal & $1552(43)$ & $1343(43.9)$ & $29(38.1)$ & \\
\hline Overweight & 1067 (29.6) & 903 (29.5) & $164(29.9)$ & \\
\hline Obese & 708 (19.6) & $573(18.7)$ & $135(24.6)$ & \\
\hline \multicolumn{5}{|l|}{ Number of medications taken } \\
\hline None & $43(1.2)$ & $8(0.3)$ & $35(6.4)$ & \multirow{4}{*}{$<0.001$} \\
\hline $1-2$ & $2175(60.3)$ & $1940(63.4)$ & $235(42.9)$ & \\
\hline $3-4$ & $1152(32)$ & $934(30.5)$ & $218(39.8)$ & \\
\hline$>4$ & $236(6.5)$ & $176(5.8)$ & $60(11.0)$ & \\
\hline Length of hospital stay (days) & $27.4 \pm 24.0$ & $27.2 \pm 23.9$ & $27.9 \pm 24.7$ & 0.533 \\
\hline Time to assessment (days) & $664.1 \pm 522.2$ & $677 \pm 519.8$ & $592.2 \pm 530.2$ & $<0.001$ \\
\hline
\end{tabular}

Data are presented as mean \pm standard deviation or $N(\%)$ unless otherwise indicated

$A E D$ anti-epileptic drug, $B M I$ body mass index 
Logit link function and log link functions were used for mean and precision model components (see the ESM).

SAS version 9.4 (SAS Institute, Cary, NC, USA) and R version 3.6.3 were used for all analyses. All effect estimates are presented with $95 \%$ confidence intervals (CIs). Significance was assumed with a $p$ value of $<0.01$.

\subsection{Sensitivity Analyses}

To examine the robustness of our results, two sensitivity analyses were considered. Both related to the exclusion criteria of our selected samples (Fig. 1A). First, stroke patients who received medication to manage other neurological conditions were included. The second included patients who had AED exposure only before stroke.

\section{Results}

We identified 3606 patients who experienced an ischaemic stroke in New Zealand from January 2012 to December 2017 who fulfilled the inclusion criteria. Among them, 548 (15\%) were exposed to an AED after stroke or both before and after stroke diagnosis. The remaining 3058 (85\%) stroke patients did not receive an AED and were thus unexposed (Table 1).

\subsection{Background Characteristics}

Patients in the AED-exposed group were generally younger than unexposed patients; $80.6 \%$ of the patients in the unexposed group were aged $\geq 75$ years, compared with $69.3 \%$ in the exposed group. Although both groups included more male than female patients and most patients identified as
European, patients in the AED-exposed group were less likely to be male and more likely to be Māori than those in the unexposed group.

A higher percentage of AED-exposed patients had a BMI in the overweight or obese category (54.5 vs. $48.2 \%$ ) and took three or more other centrally acting medications (50.8 vs. 36.3\%) compared to unexposed patients. While the mean time to assessment was longer for unexposed than for AEDexposed patients $(677 \pm 520$ vs. $592.2 \pm 530$ days; Fig. II in the ESM), LOS was comparable between exposed (27.9 days) and unexposed groups (27.2 days; Fig. III in the ESM).

\subsection{Effect of AED Exposure on Functional Status of Stroke Patients}

AED-exposed stroke patients had higher total ADL scores (mean \pm standard deviation $27 \pm 19.6$ ) compared to AEDunexposed patients $(23.5 \pm 18.5)$. Further disaggregation of the AED exposure category revealed that the median ADL score was highest for patients exposed to AEDs only after stroke, whereas median ADL scores were comparable for unexposed patients and those exposed to AEDs both before and after stroke diagnosis (Fig. 1B).

Beta regression showed that exposure to AEDs was significantly associated with greater odds of having a higher ADL score than non-exposure (Table 2): unadjusted odds ratio (OR) 1.27 (95\% CI 1.13-1.42). After adjusting for background covariates, the OR was 1.29 (95\% CI 1.15-1.45). The estimated effects of all co-variables are shown Table II in the ESM.

For both the unadjusted and the adjusted models, overall AED exposure was associated with a decrease in precision and an increase in variance (Table 2).

Table 2 Beta regression-binary anti-epileptic drug exposure

\begin{tabular}{|c|c|c|c|c|}
\hline \multirow[t]{2}{*}{ AED exposure } & \multicolumn{2}{|l|}{ Unadjusted effect } & \multicolumn{2}{|l|}{ Adjusted effect } \\
\hline & OR estimate & $p$ value & OR estimate & $p$ value \\
\hline \multicolumn{5}{|l|}{$\operatorname{Mean}_{\operatorname{model}}^{\mathrm{a}}(n=3606)$} \\
\hline Unexposed & Reference & & Reference & \\
\hline Exposed & $1.27(1.13-1.42)$ & $<0.001$ & $1.29(1.15-1.45)$ & $<0.001$ \\
\hline \multirow[t]{2}{*}{${\text { Precision } \text { model }^{\mathrm{a}}(n=3606)}$} & \multicolumn{2}{|l|}{ Unadjusted effect } & \multicolumn{2}{|l|}{ Adjusted effect } \\
\hline & Estimate & $p$ value & Estimate & $p$ value \\
\hline Unexposed & Reference & & Reference & \\
\hline Exposed & $-0.17(-0.27$ to -0.07$)$ & 0.001 & $-0.15(-0.26$ to -0.05$)$ & 0.004 \\
\hline
\end{tabular}

Figures in parentheses are $95 \%$ confidence intervals

$A E D$ anti-epileptic drug, $O R$ odds ratio

${ }^{a}$ The mean and precision components of the model were adjusted for age, sex, ethnicity, body mass index, length of hospital stay, time to assessment, and other medications. All centrally acting medications were considered in the mean model; the precision model used non-steroidal antiinflammatory drugs, anti-psychotics, and anti-nausea medications 
Table 3 Beta regression - three-category anti-epileptic drug exposure

\begin{tabular}{|c|c|c|c|c|}
\hline \multirow[t]{2}{*}{ Exposure } & \multicolumn{2}{|l|}{ Unadjusted effect } & \multicolumn{2}{|l|}{ Adjusted effect } \\
\hline & OR estimate & $p$ value & OR estimate & $p$ value \\
\hline \multicolumn{5}{|l|}{$\operatorname{Mean}_{\operatorname{model}}^{\mathrm{a}}(n=3606)$} \\
\hline Unexposed & Reference & & Reference & \\
\hline After only & $1.61(1.37-1.88)$ & $<0.001$ & $1.52(1.31-1.78)$ & $<0.001$ \\
\hline Both before and after & $0.99(0.84-1.16)$ & 0.919 & $1.09(0.93-1.27)$ & 0.310 \\
\hline \multirow[t]{2}{*}{ Exposure } & \multicolumn{2}{|l|}{ Unadjusted effect } & \multicolumn{2}{|l|}{ Adjusted effect } \\
\hline & Estimate & $p$ value & Estimate & $p$ value \\
\hline \multicolumn{5}{|c|}{ Precision model $^{\mathrm{a}}(n=3606)$} \\
\hline Unexposed & Reference & & Reference & \\
\hline After only & $-0.12(-0.26$ to 0.01$)$ & 0.065 & $-0.18(-0.32$ to -0.04$)$ & 0.013 \\
\hline Both before and after & $-0.13(-0.27$ to 0.01$)$ & 0.068 & $-0.08(-0.29$ to 0.20$)$ & 0.277 \\
\hline
\end{tabular}

Figures in parentheses are $95 \%$ confidence intervals

$A E D$ anti-epileptic drug, $O R$ odds ratio

${ }^{a}$ The mean and precision components of the model were adjusted for age, sex, ethnicity, body mass index, length of hospital stay, time to assessment, and other medications. All centrally acting medications were considered in the mean model; the precision model used non-steroidal antiinflammatory drugs, anti-psychotics, and anti-nausea medications

Table 4 Sensitivity analysis 1

\begin{tabular}{|c|c|c|c|c|}
\hline \multirow[t]{2}{*}{ AED exposure status } & \multicolumn{2}{|l|}{ Unadjusted effect } & \multicolumn{2}{|l|}{ Adjusted effect } \\
\hline & OR estimate & $p$ value & OR estimate & $p$ value \\
\hline \multicolumn{5}{|l|}{ Binary AED exposure } \\
\hline Unexposed & Reference & & Reference & \\
\hline Exposed & $1.25(1.12-1.39)$ & $<0.001$ & $1.31(1.18-1.47)$ & $<0.001$ \\
\hline \multicolumn{5}{|c|}{ Three-category AED exposure } \\
\hline Unexposed & Reference & & Reference & \\
\hline After only & $1.61(1.39-1.87)$ & $<0.001$ & $1.55(1.34-1.80)$ & $<0.001$ \\
\hline Both before and after & $0.97(0.84-1.13)$ & 0.728 & $1.10(0.95-1.28)$ & 0.219 \\
\hline
\end{tabular}

Mean model $(n=3878)$. Figures in parentheses are $95 \%$ confidence intervals. The mean and precision components of the model were adjusted for age, sex, ethnicity, BMI status, length of stay, time to assessment, and other medications. All centrally acting medications were considered in the mean model

$A E D$ anti-epileptic drug, $O R$ odds ratio

AED exposure was further categorised into only after and both before and after stroke (Table 3). The unadjusted and adjusted OR for AED exposure only after stroke was 1.61 (95\% CI 1.37-1.88) and 1.52 (95\% CI 1.31-1.78), respectively (Table 3 ). In both situations, stroke patients with AED exposure only after stroke had significantly greater odds of having a higher ADL score than unexposed patients.

Similarly, the unadjusted and adjusted OR for AED exposure before and after stroke was 0.99 (95\% CI 0.84-1.16) and 1.09 (95\% CI 0.93-1.27), respectively (Table 3).

\subsection{Sensitivity Analyses}

In the first sensitivity analysis, stroke patients who received medications to manage Parkinson's disease, Huntington's disease, Meniere's disease, dementia, or chemotherapy-associated nausea were included, increasing the sample size from 3606 to 3878 (Table 4).

The second sensitivity analysis included stroke patients who had exposure to AEDs only before their stroke diagnosis. After adding 60 such patients, our sample size increased to 3938 (Table 5). Inclusion of these patients did not change the effect estimates, indicating that the primary analyses were robust even though the inclusion criteria were flexible. 
Table 5 Sensitivity analysis 2

\begin{tabular}{|c|c|c|c|c|}
\hline \multirow[t]{2}{*}{ AED exposure status } & \multicolumn{2}{|l|}{ Unadjusted effect } & \multicolumn{2}{|l|}{ Adjusted effect } \\
\hline & OR estimate & $p$ value & OR estimate & $p$ value \\
\hline \multicolumn{5}{|l|}{ Binary AED exposure } \\
\hline Unexposed & Reference & & Reference & \\
\hline Exposed & $1.28(1.15-1.43)$ & $<0.001$ & $1.31(1.18-1.46)$ & $<0.001$ \\
\hline \multicolumn{5}{|c|}{ Three-category AED exposure } \\
\hline Unexposed & Reference & & Reference & \\
\hline After only & $1.60(1.37-1.86)$ & $<0.001$ & $1.54(1.33-1.79)$ & $<0.001$ \\
\hline Both before and after & $1.01(0.86-1.17)$ & 0.94 & $1.09(0.94-1.26)$ & 0.271 \\
\hline
\end{tabular}

Mean model $(n=3938)$. Figures in parentheses are $95 \%$ confidence intervals. The mean and precision components of the model were adjusted for age, sex, ethnicity, body mass index status, length of hospital stay, time to assessment, and other medications. All centrally acting medications were considered in the mean model

$A E D$ anti-epileptic drug, $O R$ odds ratio

\section{Discussion}

The current study examined the relationship between exposure to AEDs and post-stroke functional status in a cohort of New Zealand patients who experienced an ischaemic stroke. Stroke patients exposed to AEDs had 1.3-times higher odds of having poorer functional status than unexposed stroke patients. The difference in total ADL scores between exposed and unexposed patients was similar to that between patients requiring supported care and those living at home. This suggests AED exposure has a sufficiently large impact on functional status to affect independence. Our findings support the prevailing hypothesis in the literature that AED exposure has a detrimental effect on stroke outcome [22].

The timeframe of exposure to AEDs in the current study allowed discrimination of different clinical scenarios. Exposure before and after stroke indicated patients with epilepsy who subsequently developed a stroke (post-epilepsy stroke), whereas exposure only after stroke indicated patients who developed epilepsy as a result of their stroke (post-stroke epilepsy). We found the timeframe of exposure to AEDs had a significant impact on functional status. The odds of having poorer post-stroke functional status were significantly higher (1.5 times) in patients exposed to AEDs only after stroke than in stroke patients who were exposed to AEDs both before and after stroke and unexposed patients.

The absence of higher ADL scores in patients with postepilepsy stroke does not support a direct association between AED exposure and poor functional status, and several questions persist. It remains unclear whether there were inherent differences in stroke type between populations in the current study, whether effective management of epilepsy prior to stroke conferred benefit, and whether exposure to particular AEDs following stroke was detrimental.
Attributing poorer functional status to specific AEDs was beyond the scope of the current analysis. Exploratory analysis showed that prescribing patterns were broadly comparable across exposure timeframes and predominantly involved first- and second-generation AEDs.

The strengths of this study include the large size of the cohort assembled from population-level health administrative data, allowing our results to be generalizable to the wider New Zealand population. Using beta regression to model appropriately the skewed distribution of ADL scores is novel, and the inclusion of sensitivity analyses further validates our findings.

Our study has a few limitations applicable to any observational research using health administrative data. The covariable 'exposure to other centrally acting medications' created from the Pharmaceutical Collection does not contain inpatient hospital prescriptions or over-the-counter medication. Medications included in the AED variable can be prescribed for conditions other than epilepsy, and the absence of dose information makes it difficult to confirm the prescribing indication and the impact of conditions other than epilepsy on functional status. Although other neurological conditions were inferred by prescribing of particular medications, our data did not contain information on smoking status or comorbidities such as hypertension and diabetes that could influence patient outcomes. NHI-linked patient data were not available in the New Zealand Thrombolysis Register prior to 2018 and could not be combined with the health administrative data for this cohort. However, the current national thrombolysis rate in NZ is approximately $7 \%$ [23], implying that the majority of the patients in the current study did not receive thrombolysis. Consequently, we observed no difference in LOS between AED-exposed and -unexposed patients, suggesting that poorer functional status in AED-exposed patients was not related to greater initial stroke severity. Although we recognise that other 
factors besides stroke severity contribute to LOS, including socioeconomic status [24], LOS remains a reliable predictor of and reasonable proxy for stroke severity $[19,25]$. As mentioned, stroke location was unknown for this cohort, and it is possible that the higher incidence of cortical infarcts in patients who developed post-stroke epilepsy led to more profound motor deficits and ultimately greater disability in performing ADLs.

\section{Conclusion}

We used population-based health administrative data to demonstrate that AED exposure was associated with poorer long-term functional status after stroke. Further, we demonstrated that functional status was significantly poorer in patients who developed epilepsy after stroke than in patients with epilepsy who subsequently had a stroke.

Although this study identified a patient population likely to require additional support after stroke, it remains unclear whether it was the AEDs, the development of epilepsy, or a combination of these factors that contributed to poorer functional status. Future studies should consider a detailed analysis of AED prescribing patterns both before and after stroke to determine how specific generations or mechanistic classes of AEDs influence functional status. Understanding the impact of these treatments on stroke recovery may enable healthcare professionals to reduce severe stroke-related deficits.

Supplementary Information The online version contains supplementary material available at https://doi.org/10.1007/s40801-021-00280-5.

Acknowledgements The authors thank Dr Bruce Russell for assistance with medicine classification.

\section{Declarations}

Funding No sources of funding were used to conduct this study or prepare this manuscript.

Conflict of interest Ailsa L. McGregor, Md. Rashedul Hoque, Sophia Nickel, Alesha J. Smith, and Mohammad Atiquzzaman have no conflicts of interest that are directly relevant to the content of this article.

Availability of data and material Details of how data were obtained are available from the Ministry of Health (https://www.health.govt.nz/ nz-health-statistics/national-collections-and-surveys/collections/natio nal-minimum-dataset-hospital-events).

Code availability Custom code will be made available on request.

Ethics approval The Human Ethics Committee at the University of Otago, New Zealand, reviewed and approved this study (reference: HD17/065).
Consent The National Ethics Advisory Committee's ethical guidelines permits the re-use and publication of de-identified Ministry of Health data for research with a written consent waiver.

Author contributions ALM conceived and designed the study, supervised SN, and drafted and revised the paper. SN, MA, MRH, and AJS provided input into the study design, performed data cleaning and analysis, drafted sections of the manuscript, and reviewed paper revisions.

Data access statement Details of how data were obtained are available at the Ministry of Health (https://www.health.govt.nz/nz-health-stati stics/national-collections-and-surveys/collections/national-minimumdataset-hospital-events).

Open Access This article is licensed under a Creative Commons Attribution-NonCommercial 4.0 International License, which permits any non-commercial use, sharing, adaptation, distribution and reproduction in any medium or format, as long as you give appropriate credit to the original author(s) and the source, provide a link to the Creative Commons licence, and indicate if changes were made. The images or other third party material in this article are included in the article's Creative Commons licence, unless indicated otherwise in a credit line to the material. If material is not included in the article's Creative Commons licence and your intended use is not permitted by statutory regulation or exceeds the permitted use, you will need to obtain permission directly from the copyright holder. To view a copy of this licence, visit http://creativecommons.org/licenses/by-nc/4.0/.

\section{References}

1. Zou S, Wu X, Zhu B, Yu J, Yang B, Shi J. The pooled incidence of post-stroke in 102008 patients. Top Stroke Rehabil. 2015;22(6):460-7.

2. Conrad J, Pawlowski M, Dogan M, Kovac S, Ritter MA, Evers S. Seizures after cerebrovascular events: risk factors and clinical features. Seizure. 2013;22(4):275-82.

3. Zhao Y, Li X, Zhang K, Tong T, Cui R. The progress of epilepsy after stroke. Curr Neuropharmacol. 2018;16(1):71-8.

4. Holtkamp M, Beghi E, Benninger F, Kalviainen R, Rocamora R, Christensen $\mathrm{H}$, et al. European Stroke Organisation guidelines for the management of post-stroke seizures and epilepsy. Eur Stroke J. 2017;2(2):103-15.

5. Xu MY. Poststroke seizure: optimising its management. Stroke Vasc Neurol. 2019;4(1):48-56.

6. Rowe AS, Goodwin H, Brophy GM, Bushwitz J, Castle A, Deen $\mathrm{D}$, et al. Seizure prophylaxis in neurocritical care: a review of evidence-based support. Pharmacotherapy. 2014;34(4):396-409.

7. Messe SR, Sansing LH, Cucchiara BL, Herman ST, Lyden PD, Kasner SE, et al. Prophylactic antiepileptic drug use is associated with poor outcome following ICH. Neurocrit Care. 2009;11(1):38-44.

8. Oros MM, Smolanka VI, Sofilkanich NV, Borovik OI, Luts VV, Andrukh PG. Epilepsy after ishemic stroke: is it worth administering anticonvulsants after the first attack? Wiad Lek. 2018;71(2 pt 1):269-72.

9. Cramer SC. Drugs to enhance motor recovery after stroke. Stroke. 2015;46(10):2998-3005.

10. Viale L, Catoira NP, Di Girolamo G, Gonzalez CD. Pharmacotherapy and motor recovery after stroke. Expert Rev Neurother. 2018;18(1):65-82.

11. Yang Y, Shuaib A, Li Q, Siddiqui MM. Neuroprotection by delayed administration of topiramate in a rat model of middle cerebral artery embolization. Brain Res. 1998;804(2):169-76. 
12. Brailowsky S, Knight RT, Efron R. Phenytoin increases the severity of cortical hemiplegia in rats. Brain Res. 1986;376(1):71-7.

13. Ryvlin P, Montavont A, Nighoghossian N. Optimizing therapy of seizures in stroke patients. Neurology. 2006;67(12 Suppl 4):S3-9.

14. Brandt C, Gastens AM, Sun M, Hausknecht M, Löscher W. Treatment with valproate after status epilepticus: effect on neuronal damage, epileptogenesis, and behavioral alterations in rats. Neuropharmacology. 2006;51(4):789-804.

15. Wang Z, Tsai LK, Munasinghe J, Leng Y, Fessler EB, Chibane $F$, et al. Chronic valproate treatment enhances postischemic angiogenesis and promotes functional recovery in a rat model of ischemic stroke. Stroke. 2012;43(9):2430-6.

16. Morris JN, Fries BE, Morris SA. Scaling ADLs within the MDS. J Gerontol Ser A. 1999;54(11):M546-53.

17. Alawieh A, Zhao J, Feng W. Factors affecting post-stroke motor recovery: Implications on neurotherapy after brain injury. Behav Brain Res. 2018;340:94-101.

18. Ministry of Health. Tier 1 statistics 2016/17. New Zealand Health Survey.
19. Appelros P. Prediction of length of stay for stroke patients. Acta Neurol Scand. 2007;116(1):15-9.

20. Kang JH, Bae HJ, Choi YA, Lee SH, Shin HI. Length of hospital stay after stroke: a Korean nationwide study. Ann Rehabil Med. 2016;40(4):675-81.

21. Goldstein LB. Potential effects of common drugs on stroke recovery. Arch Neurol. 1998;55(4):454-6.

22. Tanaka T, Ihara M. Post-stroke epilepsy. Neurochem Int. 2017;107:219-28.

23. Liu Q, Ranta AA, Abernethy G, Barber PA. Trends in New Zealand stroke thrombolysis treatment rates. N Z Med J. 2017;130(1453):50-6.

24. García-Rudolph A, Cegarra B, Opisso E, Tormos JM, Bernabeu M, Saurí J. Predicting length of stay in patients admitted to stroke rehabilitation with severe and moderate levels of functional impairments. Medicine. 2020;99(43):e22423.

25. Chang KC, Tseng MC, Weng HH, Lin YH, Liou CW, Tan TY. Prediction of length of stay of first-ever ischemic stroke. Stroke. 2002;33(11):2670-4. 